\title{
PARTIAL TENSOR-PRODUCT FUNCTORS AND CROSSED-PRODUCT FUNCTORS
}

\author{
JULIAN KRANZ AND TIMO SIEBENAND
}

\begin{abstract}
For a given discrete group $G$, we apply results of Kirchberg on exact and injective tensor products of $C^{*}$-algebras to give an explicit description of the minimal exact correspondence crossed-product functor and the maximal injective crossed-product functor for $G$ in the sense of Buss, Echterhoff and Willett. In particular, we show that the former functor dominates the latter.
\end{abstract}

\section{INTRODUCTION}

A fruitful approach to construct examples of $C^{*}$-algebras is to complete *algebras with respect to certain $C^{*}$-norms. For instance, if $G \curvearrowright A$ is an action of a discrete group on a $C^{*}$-algebra, one can complete the algebraic crossed product $A \rtimes_{\text {alg }} G$ to get the maximal crossed product $A \rtimes G$ or the reduced crossed product $A \rtimes_{r} G$.

In the last decade, there has been an increasing interest in exotic completions of $A \rtimes_{\text {alg }} G$, i.e. completions which strictly lie between the maximal and reduced completion. One important motivation comes from the Baum-Connes conjecture with coefficients [BCH94] which predicts that the Baum-Connes assembly map

$$
\mu: K_{*}^{G}(\underline{E} G, A) \rightarrow K_{*}\left(A \rtimes_{r} G\right)
$$

is an isomorphism. Counterexamples to the conjecture were constructed in HLS02] by exploiting non-exactness of the functor $-\rtimes_{r} G$ for certain groups $G$. Later, in [BGW16] it was suggested to modify the conjecture by replacing the reduced crossed product with the minimal exact Morita compatible crossed product. This modification strictly enlarges the class of actions $G \curvearrowright A$ for which the conjecture is known to hold and does not change the statement of the conjecture for exact groups. Other motivations to study exotic crossed-product functors come from a$T$-menability and property $(T)$ BG13] or from non-commutative duality KLQ13, KLQ16, KLQ18,BE14.

General exotic crossed-product functors and their properties were studied systematically by Buss, Echterhoff and Willett BEW17, BEW18a, BEW18b, BEW20a, BEW20b. They introduced the minimal exact crossed-product functor $-\rtimes_{\mathcal{E}} G$, the minimal exact correspondence crossed-product functor $-\rtimes_{\mathcal{E}_{\mathfrak{E} \text { or }}} G$ (which agrees with the minimal exact Morita compatible crossed-product functor of [BGW16] for

2010 Mathematics Subject Classification. 46L55 (Primary) 46M15; 46L80 (Secondary).

Key words and phrases. $C^{*}$-algebras, exotic crossed products, tensor products.

Both authors were funded by the Deutsche Forschungsgemeinschaft (DFG, German Research Foundation) - Project-ID 427320536 - SFB 1442, as well as by Germany's Excellence Strategy EXC 2044 390685587, Mathematics Münster: Dynamics-Geometry-Structure. 
separable $G$ - $C^{*}$-algebras [BEW18a, Cor. 8.13]) and the maximal injective crossedproduct functor $-\rtimes_{\text {inj }} G$. All these functors agree with the reduced crossed product for exact groups, but their interrelations for non-exact groups are still unclear. In particular, it is unclear whether or not $-\rtimes_{\mathcal{E}} G$ and $-\rtimes_{\mathcal{E}_{\mathfrak{E} \text { orr }}} G$ agree. A positive answer to this question would imply that the "new" Baum-Connes conjecture of [BGW16] agrees with the old conjecture of [BCH94] for complex coefficients $A=\mathbb{C}$. The aim of this article is to provide an explicit description of $-\rtimes_{\text {inj }} G$ and $-\rtimes_{\mathcal{E}_{\mathfrak{E}^{\circ} \text { r }}} G$. We hope that the interplay of the universal properties and the explicit descriptions of these functors turn out useful in the future. Our main ingredient is the following construction by Kirchberg:

Theorem A ([Kir95]). There is a tensor-product functor $-\otimes_{i, \varepsilon}-$ satisfying the following properties:

(1) For every $C^{*}$-algebra $A, A \otimes_{i, \varepsilon}-$ is the minimal exact partial tensor-product functor for $A$.

(2) For every $C^{*}$-algebra $B,-\otimes_{i, \varepsilon} B$ is the maximal injective partial tensorproduct functor for $B$.

In particular, $-\otimes_{i, \varepsilon}-$ is the unique tensor-product functor which is injective in the first variable and exact in the second variable. Furthermore, $-\otimes_{i, \varepsilon}-$ is functorial for completely positive maps in both variables.

In terms of Kirchberg's tensor product, we can describe $-\rtimes_{\text {inj }} G$ and $-\rtimes_{\mathcal{E}_{\mathfrak{C}^{\circ} \text { or }}} G$ as follows:

Theorem B (Theorem 4.3). Let $G$ be a discrete group and let $A$ be a $G$-C ${ }^{*}$-algebra. Then there are injective $*$-homomorphisms

(1) $A \rtimes_{\text {inj }} G \hookrightarrow\left(A \rtimes_{r} G\right) \otimes_{i, \varepsilon} C^{*}(G)$

(2) $A \rtimes_{\mathcal{E}_{\mathfrak{E} \mathfrak{o r r}}} G \hookrightarrow C_{r}^{*}(G) \otimes_{i, \varepsilon}(A \rtimes G)$

given by $a \delta_{g} \mapsto a \delta_{g} \otimes \delta_{g}$ and $a \delta_{g} \mapsto \delta_{g} \otimes a \delta_{g}$ respectively.

We obtain an even more concrete picture using $G$-injective $G$ - $C^{*}$-algebras (see p.4 for the definition). Note that $G$-injective $G$ - $C^{*}$-algebras are always unital.

Proposition $\mathbf{C}$ (Proposition 4.5). Let $G$ be a discrete group, let $A$ be a $G$ - $C^{*}$ algebra and let $I$ be a $G$-injective $G-C^{*}$-algebra (e.g. $I=\ell^{\infty}(G)$ ). Then the canonical embedding $A \hookrightarrow A \otimes_{\max } I, a \mapsto a \otimes 1$ induces an injective $*$-homomorphism

$$
A \rtimes_{\mathcal{E}_{\mathfrak{C} \text { or }}} G \hookrightarrow\left(A \otimes_{\max } I\right) \rtimes G .
$$

Note that for $I=\ell^{\infty}(G)$, this provides a positive solution to a question asked in [BEW18a, Question 9.4] and [BGW16, 8.2]. As an application, we are able to compare $-\rtimes_{\text {inj }} G$ and $-\rtimes_{\mathcal{E}_{\mathfrak{E}_{\text {or }}}} G$ :

Corollary D (Corollary 4.6). For any discrete group $G$, we have $-\rtimes_{\text {inj }} G \leq$ $-\rtimes_{\mathcal{E}_{\mathfrak{E} \mathfrak{o r r}}} G$ and $C_{\mathrm{inj}}^{*}(G)=C_{\mathcal{E}_{\mathfrak{E} \mathfrak{o r r}}}^{*}(G)$.

Thus, in order to prove that $-\rtimes_{\text {inj }} G$ and $-\rtimes_{\mathcal{E}_{\mathfrak{c}_{\text {orr }}}} G$ coincide, it would suffice to construct a crossed-product functor which is both exact and injective.

Acknowledgements. The authors would like to thank Siegfried Echterhoff for helpful discussions and comments and the anonymous referee for pointing out an error in a previous version of this article. 


\section{Preliminaries}

In this section we fix some terminology regarding crossed-product and tensorproduct functors. For definitions and basic properties of crossed products and tensor products we refer to [BO08, Wil07.

Let * Alg denote the category of $*$-algebras with $*$-homomorphisms as morphisms and let $C^{*} \mathbf{A l g}$ denote the full subcategory of $C^{*}$-algebras. For a discrete group $G$, we denote by $C^{*} \mathbf{A} \lg _{G}$ the category of $G$ - $C^{*}$-algebras with $G$-equivariant *homomorphisms.

Let $\mathcal{C}$ be a category. A functor $F^{\mu}: \mathcal{C} \rightarrow C^{*} \mathbf{A l g}$ is a $C^{*}$-completion of a functor $F: \mathcal{C} \rightarrow{ }^{*} \mathbf{A l g}$, if for every object $X$ in $\mathcal{C}, F^{\mu}(X)$ is a $C^{*}$-completion of $F(X)$ and if for every morphism $f$ in $\mathcal{C}, F^{\mu}(f)$ is an extension of $F(f)$. We define a partial order on the class of $C^{*}$-completions of a given functor $F$ by declaring $F^{\mu} \geq F^{\nu}$ if for every object $X$ in $\mathcal{C}$, the identity on $F(X)$ extends to a $*$-homomorphism $F^{\mu}(X) \rightarrow F^{\nu}(X)$.

For two $C^{*}$-algebras $A$ and $B$, we denote by $A \odot B$ the algebraic tensor product, by $A \otimes_{\max } B$ the maximal tensor product and by $A \otimes B$ the minimal tensor product. A tensor-product functor $-\otimes_{\alpha}-$ is a $C^{*}$-completion of the functor

$$
-\odot-: C^{*} \mathbf{A l g} \times C^{*} \mathbf{A l g} \rightarrow{ }^{*} \mathbf{A l g} .
$$

A partial tensor-product functor $-\otimes_{\alpha} B$ for $B$ is a $C^{*}$-completion of the functor

$$
-\odot B: C^{*} \mathbf{A l g} \rightarrow{ }^{*} \mathbf{A l g} .
$$

A partial tensor-product functor $-\otimes_{\alpha} B$ is

(1) called exact if it maps exact sequences to exact sequences;

(2) called injective if it maps injective $*$-homomorphisms to injective $*$-homomorphisms;

(3) said to have the cp-map property if for each completely positive map $\varphi: A \rightarrow$ $C$, the induced map $\varphi \odot \operatorname{id}_{B}: A \odot B \rightarrow C \odot B$ extends to a completely positive map $\varphi \otimes_{\alpha} \operatorname{id}_{B}: A \otimes_{\alpha} B \rightarrow C \otimes_{\alpha} B$.

Every (partial) tensor-product functor is dominated by the maximal tensor-product functor and dominates the minimal tensor-product functor. For a fixed $C^{*}$-algebra $B$, the functor $-\otimes_{\max } B$ is exact $[\mathrm{BO} 08$, Prop. 3.7.1] whereas the functor $-\otimes B$ is injective. Both functors have the cp-map property [BO08, Thm. 3.5.3].

For a discrete group $G$ and a $G$ - $C^{*}$-algebra $A$, we denote by $A \rtimes_{\text {alg }} G=A[G]$ the algebraic crossed product, by $A \rtimes G$ the maximal crossed product and by $A \rtimes_{r} G$ the reduced crossed product. A crossed-product functor $-\rtimes_{\mu} G$ is a $C^{*}$-completion of the algebraic crossed-product functor

$$
-\rtimes_{\text {alg }} G: C^{*} \operatorname{Alg}_{G} \rightarrow{ }^{*} \mathbf{A l g}
$$

which dominates the reduced crossed product. We write $C_{\mu}^{*}(G):=\mathbb{C} \rtimes_{\mu} G$. A crossed-product functor $-\rtimes_{\mu} G$ is

(1) called exact if it maps exact sequences to exact sequences;

(2) called injective if it maps injective $G$-equivariant *-homomorphisms to injective $*$-homomorphisms;

(3) said to have the cp-map property if for each $G$-equivariant completely positive map $\varphi: A \rightarrow B$, the induced map $\varphi \rtimes_{\text {alg }} G: A \rtimes_{\text {alg }} G \rightarrow B \rtimes_{\text {alg }} G$ extends to a completely positive map $\varphi \rtimes_{\mu} G: A \rtimes_{\mu} G \rightarrow B \rtimes_{\mu} G$. 
Every crossed-product functor is dominated by the maximal crossed product and dominates the reduced crossed product. The maximal crossed product $-\rtimes G$ is exact [Ech17, Prop. 4.8] and the reduced crossed product $-\rtimes_{r} G$ is injective EKQR06, Lem. A.16]. Both functors have the cp-map property [BEW20a, Lem. 4.8]. Every injective crossed-product functor has the cp-map property [BEW18a, Thm.4.9]. Moreover there is a maximal injective crossed-product functor $-\rtimes_{\text {inj }} G$ [BEW20b, Prop. 3.5] and a minimal exact crossed-product functor with the cp-map property $-\rtimes_{\mathcal{E}_{\mathfrak{E} \mathfrak{r} r}} G$ [BEW18a, Cor. 8.8].

Remark 2.1. It was shown in [BEW18a, Thm. 4.9] that a crossed-product functor has the cp-map property if and only if it extends to a functor on the $G$ equivariant correspondence category $\operatorname{Corr}(G)$ as defined in BEW18a, Def. 4.4]. Therefore crossed-product functors with the cp-map property are called correspondence crossed-product functors in [BEW18a] and $-\rtimes_{\mathcal{E}_{\mathfrak{E} \mathfrak{o r r}}} G$ is called the minimal exact correspondence crossed-product functor. One can prove a similar characterization for partial tensor-product functors.

A $G$ - $C^{*}$-algebra $I$ is called $G$-injective if for every injective $G$-equivariant *homomorphism $\iota: A \hookrightarrow B$ and every $G$-equivariant completely positive contractive map $\varphi: A \rightarrow I$, there is a $G$-equivariant completely positive contractive map $\bar{\varphi}: B \rightarrow I$ such that $\bar{\varphi} \circ \iota=\varphi$. We say that $\bar{\varphi}$ extends $\varphi$ along $\iota$. In this case $I$ is unital since there exists a conditional expectation from the unitization $\tilde{I}$ onto $I$.

\section{EXACT AND INJECTIVE TENSOR-PRODUCT FUNCTORS}

In this section we give a detailed proof of a theorem that was stated in Kir95 for convenience of the reader. We need a folklore lemma.

Lemma 3.1. Let

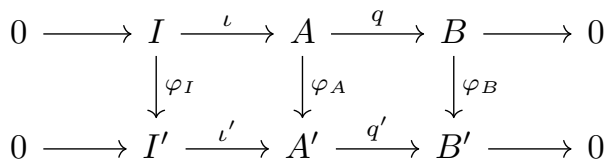

be a commutative diagram of $C^{*}$-algebras and $*$-homomorphisms. Assume that $\iota$ is an ideal inclusion, that the lower row is exact, and that the vertical maps are non-degenerate inclusions. Then we have $\operatorname{ker} q \subseteq \operatorname{im}(\iota)$.

Proof. Let $x \in \operatorname{ker} q$. By exactness, we find $y \in I^{\prime}$ such that $\iota^{\prime}(y)=\varphi_{A}(x)$. Let $\left(e_{\lambda}\right)_{\lambda}$ be an approximate unit for $I$. Since $\varphi_{I}$ is non-degenerate, $\left(\varphi_{I}\left(e_{\lambda}\right)\right)_{\lambda}$ is an approximate unit for $I^{\prime}$ and thus $\left\|\varphi_{I}\left(e_{\lambda}\right) y-y\right\| \rightarrow 0$. We obtain $\left\|\varphi_{A}\left(\iota\left(e_{\lambda}\right) x-x\right)\right\|=$ $\left\|\iota^{\prime}\left(\varphi_{I}\left(e_{\lambda}\right) y-y\right)\right\| \rightarrow 0$. This implies $\left\|\iota\left(e_{\lambda}\right) x-x\right\| \rightarrow 0$ because $\varphi_{A}$ is isometric and therefore $x \in \operatorname{im}(\iota)$ since $\iota$ is an ideal inclusion.

Theorem 3.2 ([Kir95]). There is a tensor-product functor $-\otimes_{i, \varepsilon}-$ satisfying the following properties:

(1) For every $C^{*}$-algebra $A, A \otimes_{i, \varepsilon}-$ is the minimal exact partial tensor-product functor for $A$.

(2) For every $C^{*}$-algebra $B,-\otimes_{i, \varepsilon} B$ is the maximal injective partial tensorproduct functor for $B$. 
In particular, $-\otimes_{i, \varepsilon}-$ is the unique tensor-product functor which is injective in the first variable and exact in the second variable. Furthermore, $-\otimes_{i, \varepsilon}-$ has the cp-property in both variables.

Proof. Let $A$ and $B$ be $C^{*}$-algebras and let $\iota: A \hookrightarrow \mathcal{B}(H)$ be an embedding into the bounded operators on a Hilbert space. We define

$$
A \otimes_{i, \varepsilon} B:=\iota \otimes \operatorname{id}_{B}\left(A \otimes_{\max } B\right) \subseteq \mathcal{B}(H) \otimes_{\max } B .
$$

To show that $-\otimes_{i, \varepsilon}-$ has the desired properties, we verify the following claims:

Claim 1. Up to canonical isomorphism, the definition of $A \otimes_{i, \varepsilon} B$ is independent of $\iota$.

Let $\iota^{\prime}: A \hookrightarrow \mathcal{B}\left(H^{\prime}\right)$ be another embedding. Then by Arveson's extension theorem there exist completely positive contractive maps $\Psi: \mathcal{B}(H) \rightarrow \mathcal{B}\left(H^{\prime}\right)$ extending $\iota^{\prime}$ along $\iota$ and $\Phi: \mathcal{B}\left(H^{\prime}\right) \rightarrow \mathcal{B}(H)$ extending $\iota$ along $\iota^{\prime}$. Then $\Psi \otimes_{\max } \operatorname{id}_{B}$ and $\Phi \otimes_{\max } \operatorname{id}_{B}$ restrict to mutually inverse $*$-isomorphisms

$$
\iota \otimes \operatorname{id}_{B}\left(A \otimes_{\max } B\right) \cong \iota^{\prime} \otimes \operatorname{id}_{B}\left(A \otimes_{\max } B\right) .
$$

Claim 2. $A \otimes_{i, \varepsilon} B$ is functorial for completely positive maps in both variables.

Functoriality for completely positive maps in $B$ follows immediately from the definition. To see functoriality in $A$, let $\varphi: A_{1} \rightarrow A_{2}$ be a completely positive map and let $\iota_{j}: A_{j} \hookrightarrow \mathcal{B}\left(H_{j}\right), j=1,2$ be embeddings. Let $\Psi: \mathcal{B}\left(H_{1}\right) \rightarrow \mathcal{B}\left(H_{2}\right)$ be a completely positive map extending $\iota_{2} \circ \varphi$ along $\iota_{1}$. Then $\Psi \otimes_{\max } \operatorname{id}_{B}$ restricts to a completely positive map $A_{1} \otimes_{i, \varepsilon} B \rightarrow A_{2} \otimes_{i, \varepsilon} B$ extending the canonical map $\varphi \odot \operatorname{id}_{B}: A_{1} \odot B \rightarrow A_{2} \odot B$.

Claim 3. The functor $-\otimes_{i, \varepsilon} B$ is the maximal injective partial tensor-product functor for $B$.

Let $\varphi: A_{1} \hookrightarrow A_{2}$ be an injective $*$-homomorphism and let $\iota: A_{2} \hookrightarrow \mathcal{B}(H)$ be an embedding. Then $\varphi \circ \iota: A_{1} \hookrightarrow \mathcal{B}(H)$ is an embedding too. Inserting this embedding into (11) shows that $\varphi \otimes \operatorname{id}_{B}: A_{1} \otimes_{i, \varepsilon} B \rightarrow A_{2} \otimes_{i, \varepsilon} B$ is isometric and therefore injective. Now let $-\otimes_{\alpha} B$ be another injective partial tensor-product functor for $B$ and let $A \hookrightarrow \mathcal{B}(H)$ be an embedding. Then the canonical quotient map $\mathcal{B}(H) \otimes_{\max } B \rightarrow \mathcal{B}(H) \otimes_{\alpha} B$ restricts to a quotient map $A \otimes_{i, \varepsilon} B \rightarrow A \otimes_{\alpha} B$. Thus, $-\otimes_{i, \varepsilon} B$ is maximal.

Claim 4. The functor $A \otimes_{i, \varepsilon}-$ is exact.

Let $0 \rightarrow I \stackrel{\iota}{\rightarrow} B \stackrel{\pi}{\rightarrow} Q \rightarrow 0$ be an exact sequence of $C^{*}$-algebras. Assume first that $A$ is unital and choose a unital embedding $A \hookrightarrow \mathcal{B}(H)$. Then the upper row of the diagram

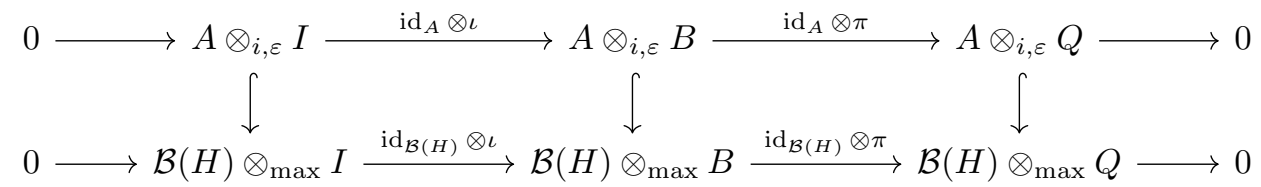


is exact by Lemma 3.1. Now assume that $A$ is not unital and denote by $\tilde{A}$ its unitization. By the above, the middle and lower row of the diagram

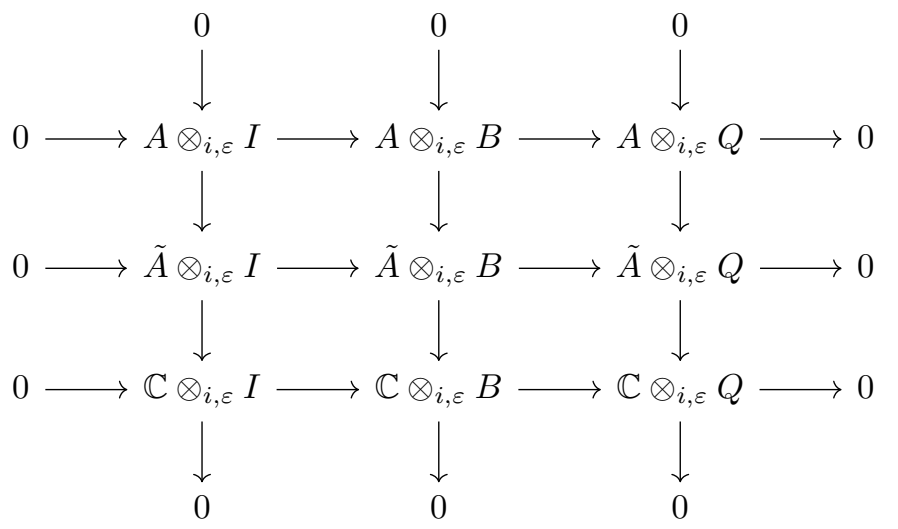

are exact. Since the extension $0 \rightarrow A \rightarrow \tilde{A} \rightarrow \mathbb{C} \rightarrow 0$ splits, the columns of (2) are exact as well. Now exactness of the upper row of (2) follows from the $3 \times 3$-Lemma.

Claim 5. The functor $A \otimes_{i, \varepsilon}-$ is the minimal exact partial tensor-product functor.

Let $A \otimes_{\alpha}$ - be another exact partial tensor-product functor and fix a $C^{*}$-algebra $B$. Assume first that $B$ is unital and pick a surjective $*$-homomorphism $C^{*}\left(F_{X}\right) \rightarrow$ $B$ where $F_{X}$ denotes the free group on a set $X$ of unitaries generating $B$. Denote by $I$ the kernel of $C^{*}\left(F_{X}\right) \rightarrow B$ and choose an embedding $\iota: A \hookrightarrow \mathcal{B}(H)$. By [Kir94, Cor. 1.2] (see also [Pis96]), there is a unique $C^{*}$-norm on $\mathcal{B}(H) \odot C^{*}\left(F_{X}\right)$. In particular, we have a canonical $*$-homomorphism

$$
A \otimes_{\alpha} C^{*}\left(F_{X}\right) \rightarrow A \otimes C^{*}\left(F_{X}\right) \stackrel{\iota \otimes \mathrm{id}}{\longrightarrow} \mathcal{B}(H) \otimes C^{*}\left(F_{X}\right)=\mathcal{B}(H) \otimes_{\max } C^{*}\left(F_{X}\right)
$$

mapping $A \otimes_{\alpha} I$ to $\mathcal{B}(H) \otimes_{\max } I$. By exactness of both $A \otimes_{\alpha}-$ and $\mathcal{B}(H) \otimes_{\max }-$, we can fill the following diagram with the dashed $*$-homomorphism $\psi$ :

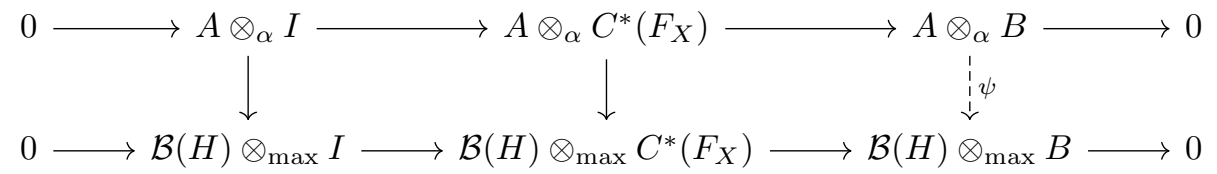

By definition, we have $\psi\left(A \otimes_{\alpha} B\right)=A \otimes_{i, \varepsilon} B$. If $B$ is a non-unital $C^{*}$-algebra, we can apply the same argument to its unitization and use exactness to produce a canonical quotient map $A \otimes_{\alpha} B \rightarrow A \otimes_{i, \varepsilon} B$. This proves maximality.

Remark 3.3. Let $F$ be a non-amenable free group and $H$ an infinite-dimensional Hilbert space. Then the flip isomorphism $\mathcal{B}(H) \odot C_{r}^{*}(F) \cong C_{r}^{*}(F) \odot \mathcal{B}(H)$ does not extend to an isomorphism $\mathcal{B}(H) \otimes_{i, \varepsilon} C_{r}^{*}(F) \cong C_{r}^{*}(F) \otimes_{i, \varepsilon} \mathcal{B}(H)$. Therefore, Kirchberg's tensor-product functor $-\otimes_{i, \varepsilon}-$ is not symmetric. Indeed, we have $C_{r}^{*}(F) \otimes_{i, \varepsilon} \mathcal{B}(H)=C_{r}^{*}(F) \otimes \mathcal{B}(H)$ since $C_{r}^{*}(F)$ is exact and $\mathcal{B}(H) \otimes_{i, \varepsilon} C_{r}^{*}(F)=$ $\mathcal{B}(H) \otimes_{\max } C_{r}^{*}(F)$ by construction. But the identity map on $\mathcal{B}(H) \odot C_{r}^{*}(F)$ does not extend to an isomorphism $\mathcal{B}(H) \otimes_{\max } C_{r}^{*}(F) \cong \mathcal{B}(H) \otimes C_{r}^{*}(F)$ since $C_{r}^{*}(F)$ does not have the local lifting property [BO08, Cor. 3.7.12, Thm. 13.1.6, Cor. 13.2.5]. 


\section{Applichtion to Crossed products}

Throughout this section, let $G$ be a discrete group. We recall a version of Fell's absorption principle from ABES21.

Proposition 4.1 ([ABES21, Prop. 2.8]). Let $-\rtimes_{\mu} G$ be a crossed-product functor with the cp-map property and let $A$ be a $C^{*}$-algebra equipped with the trivial $G$ action. Then the canonical map $A \odot C_{\mu}^{*}(G) \rightarrow A \rtimes_{\mu} G$ is injective. In particular, $A \mapsto A \otimes_{\mu} C_{\mu}^{*}(G):=A \rtimes_{\mu} G$ is a partial tensor-product functor for $C_{\mu}^{*}(G)$.

Although only stated for $\rho=\max$ in ABES21, the proof of the following lemma works verbatim for every crossed-product functor $-\rtimes_{\rho} G$ :

Lemma 4.2 ([ABES21, Lem 2.10]). Let $-\rtimes_{\mu} G$ be a crossed-product functor with the cp-map property and let $-\rtimes_{\rho} G$ be any crossed-product functor. Then for every $G-C^{*}$-algebra $A$, there is an injective *-homomorphism

$$
A \rtimes_{\mu} G \hookrightarrow\left(A \rtimes_{\rho} G\right) \otimes_{\mu} C_{\mu}^{*}(G)
$$

given by $a \delta_{g} \mapsto a \delta_{g} \otimes \delta_{g}$ for $a \in A, g \in G$.

Theorem 4.3. For every $G$ - $C^{*}$-algebra $A$, there are injective *-homomorphisms

(1) $A \rtimes_{\text {inj }} G \hookrightarrow\left(A \rtimes_{r} G\right) \otimes_{i, \varepsilon} C^{*}(G), \quad a \delta_{g} \mapsto a \delta_{g} \otimes \delta_{g}$.

(2) $A \rtimes_{\mathcal{E}_{\mathfrak{C} \mathfrak{o r} r}} G \hookrightarrow C_{r}^{*}(G) \otimes_{i, \varepsilon}(A \rtimes G), \quad a \delta_{g} \mapsto \delta_{g} \otimes a \delta_{g}$.

Proof. We first prove the statement for $-\rtimes_{\text {inj }} G$. Denote by $A \rtimes_{\alpha} G$ the image of $A \rtimes G$ in $\left(A \rtimes_{r} G\right) \otimes_{i, \varepsilon} C^{*}(G)$ under the map $a \delta_{g} \mapsto a \delta_{g} \otimes \delta_{g}$. Then $-\rtimes_{\alpha} G$ is an injective crossed-product functor and therefore $-\rtimes_{\alpha} G \leq-\rtimes_{\text {inj }} G$. On the other hand, Lemma 4.2 gives us an embedding

$$
A \rtimes_{\mathrm{inj}} G \hookrightarrow\left(A \rtimes_{r} G\right) \otimes_{\mathrm{inj}} C_{\mathrm{inj}}^{*}(G), \quad a \delta_{g} \mapsto a \delta_{g} \otimes \delta_{g}
$$

Since $-\otimes_{i, \varepsilon} C_{\mathrm{inj}}^{*}(G)$ is the maximal injective partial tensor-product functor for $C_{\text {inj }}^{*}(G)$, we have

$$
-\otimes_{i, \varepsilon} C^{*}(G) \geq-\otimes_{i, \varepsilon} C_{\mathrm{inj}}^{*}(G) \geq-\otimes_{\mathrm{inj}} C_{\mathrm{inj}}^{*}(G)
$$

and therefore $-\rtimes_{\alpha} G \geq-\rtimes_{\text {inj }} G$.

We now prove the statement for $-\rtimes_{\mathcal{E}_{\mathfrak{C} \text { o r }}} G$. Denote by $A \rtimes_{\beta} G$ the image of $A \rtimes G$ in $C_{r}^{*}(G) \otimes_{i, \varepsilon}(A \rtimes G)$ under the map $a \delta_{g} \mapsto \delta_{g} \otimes a \delta_{g}$. Then $-\rtimes_{\beta} G$ is an exact crossed-product functor with the cp-map property by Lemma 3.1 and therefore $-\rtimes_{\beta} G \geq-\rtimes_{\mathcal{E}_{\mathfrak{C}_{\mathfrak{o r} r}}} G$. On the other hand, Lemma 4.2 gives us an embedding

$$
A \rtimes_{\mathcal{E}_{\mathfrak{C} \mathfrak{o r} r}} G \hookrightarrow C_{\mathcal{E}_{\mathfrak{C} \mathfrak{o r} r}}^{*}(G) \otimes_{\mathcal{E}_{\mathfrak{C} \mathfrak{o r} r}}(A \rtimes G) .
$$

Since $C_{\mathcal{E}_{\mathfrak{C} \text { or }}}^{*}(G) \otimes_{i, \varepsilon}$ - is the minimal exact partial tensor-product functor for $C_{\mathcal{E}_{\mathfrak{C} \text { or }}}^{*}(G)$, we get

$$
C_{r}^{*}(G) \otimes_{i, \varepsilon}-\leq C_{\mathcal{E}_{\mathfrak{C o r} \mathfrak{r}}}^{*}(G) \otimes_{i, \varepsilon}-\leq C_{\mathcal{E}_{\mathfrak{C o r} \mathfrak{r}}}^{*}(G) \otimes_{\mathcal{E}_{\mathfrak{C} \mathfrak{o r} r}}-
$$

and therefore $-\rtimes_{\beta} G \leq-\rtimes_{\mathcal{E}_{\mathfrak{C} \mathfrak{o r r}}} G$.

Remark 4.4. The statement of the above theorem remains true if we replace $A \rtimes G$ by $A \rtimes_{\mathcal{E}_{\mathfrak{C}_{\mathfrak{o r} r}}} G, C^{*}(G)$ by $C_{\mathcal{E}_{\mathfrak{C} \mathfrak{o r r}}}^{*}(G), A \rtimes_{r} G$ by $A \rtimes_{\text {inj }} G$, or $C_{r}^{*}(G)$ by $C_{\text {inj }}^{*}(G)$. Indeed, the only properties of the maximal (resp. reduced) crossed product that we used in the proof were exactness (resp. injectivity) and the cp-map property. 
Proposition 4.5. Let $I$ be a $G$-injective $G-C^{*}$-algebra and let $A$ be any $G$ - $C^{*}$ algebra. Then the canonical embedding $A \hookrightarrow A \otimes \max I, a \mapsto a \otimes 1$ induces an embedding

$$
A \rtimes_{\mathcal{E}_{\mathfrak{C} \text { or }}} G \hookrightarrow\left(A \otimes_{\max } I\right) \rtimes G .
$$

Proof. Denote by $A \rtimes_{\alpha} G$ the image of $A \rtimes G$ in $\left(A \otimes_{\max } I\right) \rtimes G$ under the map $a \delta_{g} \mapsto(a \otimes 1) \delta_{g}$. Then $-\rtimes_{\alpha} G$ is an exact crossed-product functor with the cpmap property by Lemma 3.1 and therefore $-\rtimes_{\alpha} G \geq-\rtimes_{\mathcal{E}_{\mathfrak{E} \text { or }}} G$. It remains to prove the converse inequality. Consider $\mathcal{B}\left(\ell^{2}(G)\right)$ as a $G$ - $C^{*}$-algebra equipped with the conjugation action of the left regular representation $\lambda: G \rightarrow \mathcal{U}\left(\ell^{2}(G)\right)$. By $G$ injectivity, there is a $G$-equivariant unital completely positive map $\varphi: \mathcal{B}\left(\ell^{2}(G)\right) \rightarrow$ I. Consider the "untwisting isomorphism"

$$
\Psi: \mathcal{B}\left(\ell^{2}(G)\right) \otimes_{\max }(A \rtimes G) \stackrel{\cong}{\rightarrow}\left(\mathcal{B}\left(\ell^{2}(G)\right) \otimes_{\max } A\right) \rtimes G, \quad T \otimes a \delta_{g} \mapsto T \lambda_{g^{-1}} \otimes a \delta_{g}
$$

and denote by $\kappa$ the following composition of contractive maps.

$$
\begin{array}{r}
A \rtimes_{\mathcal{E}_{\mathfrak{C} \text { or } r}} G \stackrel{\text { Thm } \underline{4.3}}{\longrightarrow} C_{r}^{*}(G) \otimes_{i, \varepsilon}(A \rtimes G) \stackrel{\lambda \otimes \mathrm{id}}{\longrightarrow} \mathcal{B}\left(\ell^{2}(G)\right) \otimes_{\max }(A \rtimes G) \\
\left(I \otimes_{\max } A\right) \rtimes G \underset{(\varphi \otimes \mathrm{id}) \rtimes G}{\longleftarrow}\left(\mathcal{B}\left(\ell^{2}(G)\right) \otimes_{\max } A\right) \rtimes G
\end{array}
$$

A straightforward computation shows that $\kappa\left(a \delta_{g}\right)=(a \otimes 1) \delta_{g}$ for $a \in A$ and $g \in G$.

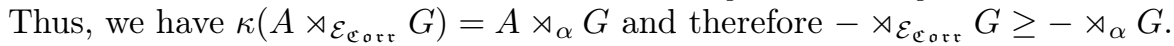

Corollary 4.6. For any discrete group $G$, we have $-\rtimes_{\text {inj }} G \leq-\rtimes_{\mathcal{E}_{\mathfrak{C} o \mathrm{r}}} G$ and $C_{\mathrm{inj}}^{*}(G)=C_{\mathcal{E}_{\mathfrak{E} \text { or r }}}^{*}(G)$.

Proof. Let $A, I$ be $G$ - $C^{*}$-algebras where $I$ is $G$-injective. The embedding $A \hookrightarrow$ $A \otimes_{\max } I$ induces an embedding $A \rtimes_{\text {inj }} G \hookrightarrow\left(A \otimes_{\max } I\right) \rtimes_{\text {inj }} G$. The first statement now follows from Proposition 4.5. The second statement follows from the same argument and the fact that $I \rtimes G=I \rtimes_{\text {inj }} G$ [BEW20b, Cor. 3.3].

\section{REFERENCES}

[ABES21] Paolo Antonini, Alcides Buss, Alexander Engel, and Timo Siebenand. Strong Novikov conjecture for low degree cohomology and exotic group $C^{*}$-algebras. Trans. Amer. Math. Soc., 374(7):5071-5093, 2021.

[BCH94] Paul Baum, Alain Connes, and Nigel Higson. Classifying space for proper actions and $K$-theory of group $C^{*}$-algebras. In $C^{*}$-algebras: 1943-1993 (San Antonio, TX, 1993), volume 167 of Contemp. Math., pages 240-291. Amer. Math. Soc., Providence, RI, 1994.

[BE14] Alcides Buss and Siegfried Echterhoff. Universal and exotic generalized fixed-point algebras for weakly proper actions and duality. Indiana Univ. Math. J., 63(6):16591701, 2014.

[BEW17] Alcides Buss, Siegfried Echterhoff, and Rufus Willett. Exotic crossed products. In Operator algebras and applications - the Abel Symposium 2015, volume 12 of Abel Symp., pages 67-114. Springer, [Cham], 2017.

[BEW18a] Alcides Buss, Siegfried Echterhoff, and Rufus Willett. Exotic crossed products and the Baum-Connes conjecture. J. Reine Angew. Math., 740:111-159, 2018.

[BEW18b] Alcides Buss, Siegfried Echterhoff, and Rufus Willett. The minimal exact crossed product. Doc. Math., 23:2043-2077, 2018.

[BEW20a] Alcides Buss, Siegfried Echterhoff, and Rufus Willett. Injectivity, crossed products, and amenable group actions. In $K$-theory in algebra, analysis and topology, volume 749 of Contemp. Math., pages 105-137. Amer. Math. Soc., [Providence], RI, 2020. 
[BEW20b] Alcides Buss, Siegfried Echterhoff, and Rufus Willett. The maximal injective crossed product. Ergodic Theory Dynam. Systems, 40(11):2995-3014, 2020.

[BG13] Nathanial P. Brown and Erik P. Guentner. New C*-completions of discrete groups and related spaces. Bull. Lond. Math. Soc., 45(6):1181-1193, 2013.

[BGW16] Paul Baum, Erik Guentner, and Rufus Willett. Expanders, exact crossed products, and the Baum-Connes conjecture. Ann. K-Theory, 1(2):155-208, 2016.

[BO08] Nathanial P. Brown and Narutaka Ozawa. $C^{*}$-algebras and finite-dimensional approximations, volume 88 of Graduate Studies in Mathematics. American Mathematical Society, Providence, RI, 2008.

[Ech17] Siegfried Echterhoff. Crossed products and the Mackey-Rieffel-Green machine. In $K$ Theory for Group $C^{*}$-Algebras and Semigroup $C^{*}$-Algebras, pages 5-79. Springer, 2017.

[EKQR06] Siegfried Echterhoff, S. Kaliszewski, John Quigg, and Iain Raeburn. A categorical approach to imprimitivity theorems for $C^{*}$-dynamical systems. Mem. Amer. Math. Soc., 180(850):viii+169, 2006.

[HLS02] N. Higson, V. Lafforgue, and G. Skandalis. Counterexamples to the Baum-Connes conjecture. Geom. Funct. Anal., 12(2):330-354, 2002.

[Kir94] Eberhard Kirchberg. Commutants of unitaries in UHF algebras and functorial properties of exactness. J. Reine Angew. Math., 452:39-77, 1994.

[Kir95] Eberhard Kirchberg. Exact $C^{*}$-algebras, tensor products, and the classification of purely infinite algebras. In Proceedings of the International Congress of Mathematicians, Vol. 1, 2 (Zürich, 1994), pages 943-954. Birkhäuser, Basel, 1995.

[KLQ13] S. Kaliszewski, Magnus B. Landstad, and John Quigg. Exotic group $C^{*}$-algebras in noncommutative duality. New York J. Math., 19:689-711, 2013.

[KLQ16] S. Kaliszewski, Magnus B. Landstad, and John Quigg. Coaction functors. Pacific J. Math., 284(1):147-190, 2016.

[KLQ18] S. Kaliszewski, Magnus B. Landstad, and John Quigg. Coaction functors, II. Pacific J. Math., 293(2):301-339, 2018.

[Pis96] Gilles Pisier. A simple proof of a theorem of Kirchberg and related results on $C^{*}$-norms. J. Operator Theory, 35(2):317-335, 1996.

[Wil07] Dana P. Williams. Crossed products of $C^{*}$-algebras, volume 134 of Mathematical Surveys and Monographs. American Mathematical Society, Providence, RI, 2007. 